\title{
Which personal, behavioural, environmental, and social factors are important in smoking cessation and relapse in male manual workers? A qualitative study in southeast England
}

Anjum Memon, Imogen Rogers, James Sidebotham, Josefin Sundin, Emma Rumsby, Samantha Parker

Department of Primary Care and Public Health, Brighton and Sussex Medical School, Brighton, UK (Prof A Memon DPhil, I Rogers PhD, J Sidebotham BMBS, J Sundin PhD, E Rumsby BMBS, S Parker BMBS)

\section{Correspondence to:}

Prof Anjum Memon, Department of Primary Care and Public Health, Brighton and Sussex Medical School, Brighton BN1 9PH, UK

a.memon@bsms.ac.uk

Background In most developed countries, the prevalence of smoking remains stubbornly high in lower socioeconomic groups. Male manual workers in England are more than twice as likely as those in managerial and professional occupations to smoke ( $26 \%$ vs $11 \%)$, find it more difficult to quit, and have the highest relapse rates. We sought to determine perceived factors in smoking cessation and relapse in this hard-to-reach group to inform the development of effective stop smoking services and public health efforts to reduce the health inequality caused by smoking.

Methods In this qualitative study in southeast England, 12 male manual workers (aged 18-65 years), who had accessed the Sussex Community NHS Trust Stop Smoking Service during the previous 12 months, were recruited into two focus groups ( $F G ~ 1, n=4 ; F G ~ 2, n=8$ ). The focus group discussions were guided by structured and prompt questions. The Brighton East Research Ethics Committee approved the study, and written informed consent was obtained from all participants.

Findings Participants identified three broad themes that influenced their smoking cessation efforts and relapse. First, personal and behavioural factors included effect of smoking on current physical fitness as a stronger motivating factor to quit than long-term health risks, risk of relapse associated with use of recreational substances (drugs, alcohol), positive impact of changing one's daily routine or habits to control smoking triggers, negative impact on children, negative social impact of "smelling of cigarettes", and perception that women have more willpower and find it easier to quit smoking. Second, environmental factors included triggering effect of stressful life events, increased risk of relapse because of the availability of packs of ten cigarettes, and the beneficial effect of a ban on point-of-sale display and smoking in pubs and bars. Third, social factors included positive impact of support from friends, family, or the local community, and negative impact of perceived greater "social acceptability" of smoking in men and "social acceptability" of smoking versus other addictions (eg, recreational drugs, heroin, alcoholism).

Interpretation This new insight informs the development of public health interventions, which need to address these social norms and attitudes in this group of disadvantaged smokers. Carefully targeted and effective campaigns with appropriate content and tone may reduce the health inequality caused by smoking.

Funding Brighton and Sussex Medical School. 
Contributors AM conceived and designed the study. SP and ER implemented the focus groups. SP, JoS, JaS, IR, and AM coded and analysed the data. SP, JoS, JaS, and IR conducted the literature search. AM and IR drafted the abstract. All authors have seen and approved the final version of the abstract for publication.

Declaration of interests: We declare no competing interests. 